\title{
DECISION SUPPORT ARSENAL USAGE FOR STRATEGIC PLANNING
}

\begin{abstract}
This paper outlines an approach to strategic planning, based on decision-making support methods. It provides a brief description of a step-by-step procedure, allowing the decision-maker to build a strategy, targeted at solution of some weakly-structured problem or at achievement of some complex goal, influenced by multiple tangible and intangible criteria. In the context of this paper a strategy is defined as the optimal way of resource distribution among specific factors or projects, which influence the main goal of a strategic plan.
\end{abstract}

Keywords: multi-criteria decision-making, prioritization, strategy, resource allocation.

\section{- Introduction}

A strategy is a long-term plan, targeted at achievement of some complex goal, influenced by a multitude of factors, both tangible and intangible. Definition of these factors and interrelations between them is a task solved through hierarchical decomposition of the goal in question. Prioritization of different factors (or criteria) is one of the most important phases of strategic planning process, as it allows the decision-maker to understand, which of the factors are crucial for the main goal of the strategy. The more important some criterion or factor is, the more resources and efforts should be focused on it in order to ensure the fastest and most efficient achievement of the main goal. While tangible factors can be numerically measured, intangible ones can only be evaluated by experts (through direct estimation in some scale or pair-wise comparisons) as shown by (Saaty, 2008). And when it comes to criterion weights, or their relative importance coefficients, expert evaluation is the only way to define them. As we can see, decision support arsenal can come into play at every phase of strategic planning process. The task of this paper is to make this process more structured, and to demonstrate how modern decision support methods and tools can further improve it.

\section{- Literature Review}

Publications by (DeFelice and Petrillo, 2013(b), 2013(b)) describe the usage of multi-criteria approach and decision analysis for specific strategic industries. A publication by (Tsyganok et al., 2015) describes a strategic planning technology, based on decision support methods, however, it is mostly focused on the benefits of usage of multiple estimation scales in the strategic planning context. The expert data-based approach to strategic planning needs to be set forth in a more explicit and clearly structured form.

\section{- Hypotheses/Objectives}

The study objective is to set forth a clear step-by-step strategic planning procedure, which can be used by strategists, operating in weakly-structured domains. As weakly structured domains are associated with multiple factors (often unquantifiable), we should define: when (at which particular stages of the process) the experts are to be addressed, what information should be obtained from them, and how this information is to be interpreted and processed.

\section{- Research Design/Methodology}

On the one hand, a strategy is, generally, a non-detailed plan. On the other, it must be robust in terms of uncertainties of the specific environment. Also, the user should be able to make the plan more specific through its decomposition down to the level of manageable factors or projects. The optimal distribution of efforts and resources between these factors or projects (with their relative weights or impacts taken into account) will be the best strategy for achieving the main goal. Based on these requirements the strategic planning procedure should include the following steps.

1) Formulation of the main goal. This task is, presumably, the responsibility of the decision-maker. 
2) Selection of experts. The task of choosing the experts, who will perform the next steps, can be placed on either the decision-maker, or the knowledge engineer. The decision-maker can be the leader of the expert group. The role of experts can be assumed by the managers of organizational units (if we are dealing with a large organization) or by external specialists from respective areas of expertise.

3) Building of the hierarchy of criteria, which influence the main goal of the strategic plan. The task of building a hierarchy is placed on the experts. Ideally, each expert should be responsible for the part of the hierarchy, which corresponds to his or her area of expertise. Again, the decomposition process must stop when the lowest level includes manageable, "atomic" projects or factors, which can be directly influenced by the decision-maker.

4) Estimation of criterion weights. Relative weight (priority) of each criterion in the hierarchy graph, i.e. its relative impact upon its "ancestor", can be defined directly or through pair-wise comparisons (Saaty 2008). To derive priorities from pair-wise comparison matrices (provided by one expert or a group of experts), one can use a combinatorial method, mentioned in (Tsyganok 2015), because it utilizes the redundancy of expert information most thoroughly, and (if necessary) provides great opportunities for consistency and compatibility improvement.

5) Calculation of the relative efficiency of projects. The projects are defined by the lowest-level nodes of the hierarchy graph. They can be directly influenced by the decision-maker. Once all the priorities are defined, the efficiency of each project can be calculated. The relative efficiency of each project is the difference between the degrees of the achievement of the main goal when the project is present in the hierarchy and when the project is excluded from it.

6) Definition of the optimal strategy. As it has been already said, The optimal strategy will be represented by the optimal distribution of resources among projects of the hierarchy.

All the aforementioned steps can be performed using the software tools available at http://dss-lab.org.ua/.

\section{- Limitations}

Resource allocation problem needs to be addressed separately, as it has some peculiarities. First, the total amount of resources is, usually, limited. Second, each project is characterized by the range of resource amounts it requires. Third, projects can be characterized by different time frames. Under the circumstances, the "easiest" (although, time- and labor-consuming) way to define the optimal resource distribution profile would be to enumerate all possible resource distribution vectors with a fixed precision, for example, using genetic algorithm.

\section{- Conclusions}

An expert data-based strategic planning technology using AHP-based approaches (such as hierarchical decomposition of goal, pair-wise comparisons, and prioritization of factors) has been suggested. Some of the aspects of the technology (data consistency and compatibility improvement, as well as resource allocation techniques) can be further improved. These tasks will be addressed in future studies.

\section{- Key References}

De Felice, F., \& Petrillo, A. (2013) Multicriteria approach for process modelling in strategic environmental management planning. International Journal of Simulation and Process Modelling, Vol. 8 (1), 6-16.

De Felice, F., \& Petrillo, A. (2013) Decision-making analysis to improve public participation in strategic energy production management. Studies in Fuzziness and Soft Computing, 305, 129-142.

Saaty, T.L. (2008) Relative measurement and its generalization in decision making. Why pair-wise comparisons are central in mathematics for the measurement of intangible factors. The Analytic Hierarchy/Network Process, Statistics and Operations Research, 102(2), 251-318.

Tsyganok, V.V, Kadenko, S.V., \& Andriichuk, O.V. (2015) Using Different Pair-wise Comparison Scales for Developing Industrial Strategies. Int. J. Management and Decision Making, 14(3), 224-250. 